\title{
LSEResearch Online
}

(

\section{Olina Efthymiadou, Jean Mossman and Panos Kanavos Differentiation of health related quality of life outcomes between five disease areas; results from an international survey of patients}

\section{Article (Accepted version) (Refereed)}

\begin{abstract}
Original citation:
Efthymiadou, Olina and Mossman, Jean and Kanavos, Panos (2018) Differentiation of health related quality of life outcomes between five disease areas; results from an international survey of patients. International Journal of Technology Assessment in Health Care, 34 (5). pp. 498-506. ISSN 0266-4623

DOI: https://doi.org/10.1017/S0266462318000557

(C) 2018 Cambridge University Press

This version available at: http://eprints.Ise.ac.uk/id/eprint/91670

Available in LSE Research Online: January 2019

LSE has developed LSE Research Online so that users may access research output of the School. Copyright (c) and Moral Rights for the papers on this site are retained by the individual authors and/or other copyright owners. Users may download and/or print one copy of any article(s) in LSE Research Online to facilitate their private study or for non-commercial research. You may not engage in further distribution of the material or use it for any profit-making activities or any commercial gain. You may freely distribute the URL (http://eprints.Ise.ac.uk) of the LSE Research Online website.

This document is the author's final accepted version of the journal article. There may be differences between this version and the published version. You are advised to consult the publisher's version if you wish to cite from it.
\end{abstract}


Title: Differentiation of Health Related Quality of Life outcomes between five disease areas; results from an international survey of patients

Short title: EQ-5D outcomes between chronic disease patients. 


\section{Authors and affiliations}

Olina Efthymiadou, MSc

Medical Technology Research Group, LSE Health

London School of Economics

Houghton Street

London WC2A 2AE

England

\section{Jean Mossman, BSc}

Medical Technology Research Group, LSE Health London School of Economics

Houghton Street

London WC2A 2AE

England

\section{Panos Kanavos, PhD}

Department of Social Policy

Medical Technology Research Group, LSE Health London School of Economics

Houghton Street

London WC2A 2AE

England

\section{$\underline{\text { Address for correspondence: }}$}

Olina Efthymiadou, MSc

Medical Technology Research Group, LSE Health London School of Economics

Houghton Street

London WC2A 2AE

England

a.efthymiadou@1se.ac.uk

\section{Panos Kanavos, PhD}

Department of Social Policy

Medical Technology Research Group, LSE Health

London School of Economics

Houghton Street

London WC2A 2AE

England

p.g.kanavos@1se.ac.uk 


\section{Abstract}

OBJECTIVES: Health Related Quality of Life (HRQoL) data generated by generic, preference-based instruments (i.e. EQ-5D) are highly demanded in health policy decisionmaking, because they allow for direct comparisons of HRQoL outcomes between disease areas. We aimed to quantify HRQoL outcomes in Breast Cancer (BC), Rheumatoid Arthritis (RA), Multiple Sclerosis (MS), Rare Cancers (RC) and Rare Disease (RD) patients and understand the patterns that differentiate HRQoL outcomes between these disease areas, and more specifically between rare and more common disease population groups. METHODS: An international, web-survey of patients measured HRQoL (EQ-5D-5L), self-perceived health (EQ-5D-5L Visual Analogue Scale) and additional QoL dimensions such as patient disability level. RESULTS: We received 675 completed responses. Average utility loss was $53.5 \%, 32.5 \%$ and $33.3 \%$ for RD, RA and MS patients respectively, in contrast to $18.6 \%$ for BC and RC patients. Statistically significant differences $(p<0.05)$ were observed between disease groups in all EQ-5D-5L domain outcomes, apart from that of "Anxiety/Depression". Severe and/or extreme problems were reported in performing usual activities for RD and RC (34\% and $13 \%$ of overall problems reported respectively), mobility for MS (18\%), pain/discomfort for RA (13\%) and anxiety/depression for BC (7\%) patients. CONCLUSIONS: We demonstrated significant differences in the dimensions that drive HRQoL outcomes between rare and more common diseases and showcased that the same EQ-5D utility may reflect very different severities depending on the patient population under investigation. Future research should examine whether outcomes in other, critical HRQoL domains not included in generic measures also highlight significant differences across disease areas. 


\section{Background}

The health-related quality of life (HRQoL) of individuals suffering from a chronic condition is often significantly impaired and this is a key concern not only for patients, their caregivers and clinicians but also for healthcare policy makers [1]. Chronic disorders often present with high socio-economic burden either due to increased mortality (i.e. malignant neoplasms being the second leading cause of death [2]), to long-term disability (i.e. in rheumatic disorders and multiple sclerosis [3]), or to high unmet treatment needs (i.e. in rare disorders, where treatment is available only for $5 \%$ of the approximately 7,000 rare diseases discovered [4]). While numerous studies have addressed the impact of chronic diseases and their treatment on HRQoL outcomes literature is still unclear about the specific dimensions of Quality of Life (QoL) that are impaired by different chronic disorders, as well as the extent to which the level of impairment on these dimensions varies between patient populations of different chronic disorders [1]. There have been very few comparisons on the impact of different chronic disorders on QoL, and even more scarce are comparisons of QoL outcomes between rare and more common disease areas [5,6]. Such comparisons are key in HRQoL research for chronic diseases since they contribute in understanding the drivers of poor outcomes in one disease area relative to another and therefore facilitate decisions on the cost-effective allocation of resources for research, health care services use and disease management across diseases [1]. In order to perform such comparisons of patient outcomes within and between disease areas, HRQoL data generated by preference based HRQoL measurement instruments are highly demanded in health policy decision making. One of the most widely used preference based instruments intended to measure and value health outcomes across a wide range of diseases and treatments is the five-dimension EuroQol (EQ-5D), which has been certified in many countries worldwide; it is commonly used in economic evaluation and technology assessment $[7,8]$ and as a standard tool in clinical studies because of its generic nature. 
Very few studies have used generic, preference based HRQoL measurement tools, namely the SF-24 [9] and the SF-36 [1] to compare outcomes between therapeutic areas but these are now out-dated and more importantly, due to their heterogeneity with respect to sample composition, sample size, instruments and procedures, it was concluded that further empirical data is needed to corroborate their findings [9]. In order to increase the body of evidence in HRQoL comparisons across chronic diseases, the aim of this paper is twofold; first to quantify health state utility outcomes across selected disease areas, namely Breast Cancer (BC), Rare Cancers (RC), Multiple Sclerosis (MS), Rheumatoid Arthritis (RA) and RD, and second to understand the patterns that differentiate HRQoL outcomes (using the fivedimension EuroQol (EQ-5D) between disease categories, within their respective disease clusters, between disease clusters and more specifically between rare and more common disease population groups. 


\section{Methods}

\section{Sample and Research design}

We conducted a retrospective, web-based survey of individuals with BC, RA, MS, RC and RD. Disease areas were selected such that they reflect a significant burden to the society but are also represent areas where EQ-5D is commonly used, such that their clinical changes are considered to be well reflected in the EQ-5D-5L instrument and used in clinical areas [10].

Participants were identified through a network of patients and patient associations' representatives, hosted by the Medical Technology Research Group at the London School of Economics (LSE). The survey was based on a multidimensional questionnaire comprising three sections which captured; a) patient demographic information and clinically relevant characteristics such as disease area and years since diagnosis, b) HRQoL, measured with the EuroQol 5-domain 5-level (EQ-5D-5L) instrument, self-perceived health (using the EQ-5D5L VAS instrument) and c) additional dimensions related to Quality of Life (QoL) outcomes such as patients' physical disability (using the Barthel Index (BI) score). Questionnaires were made available in six languages: English, German, Greek, French, Italian and Swedish.

One hundred and eighty patient associations across 48 countries, primarily from Europe but also from Australia, Brazil, China, Indonesia, Japan, Malaysia, Kyrgyzstan, Philippines, Singapore and USA were invited via e-mail to voluntarily share the questionnaire with their network of patients. Associations representing the following disease areas were invited: $\mathrm{BC}$ (e.g. Europa Donna), RA (e.g. European League Against Rheumatism - EULAR), MS (i.e. US National Multiple Sclerosis Society- NMSS), RC (i.e. Myeloma Patients Europe - MPE), and RD (e.g. Rare Connect). To ensure anonymity, questionnaire responses carried no identification information (name, address/postcode, e-mail or telephone). 
All patients were informed about the study objectives and the data confidentiality procedures in place, and were asked to provide online written informed consent to indicate their understanding of the study conditions and their agreement to participate. The study protocol was submitted to the LSE Research Ethics Committee and the committee determined that this study was exempt from formal human subjects ethics review because the data collected and used for analysis were anonymous and carried no identification information.

Preceding the actual survey questions, an online information sheet not only described the objectives of the survey and processes related to data confidentiality, but also provided a brief description of the EQ-5D-5L tool and its utilisation in HRQoL measurement. The survey was hosted online on Qualtrics ${ }^{\circledR}$ software under an LSE-verified account and remained open between June and August 2015.

\section{Statistical analyses}

The IBM Statistical Package for Social Sciences (SPSS) version 21 was used to generate descriptive statistics (mean, median and standard deviation) and assess the statistical significance of differences in HRQoL outcomes between disease areas. HSUVs, VAS, and BI scores were all treated as continuous variables. These were assessed for normality using the Kolmogorov-Smirnov test and were found to deviate significantly from the normal distribution $(\mathrm{p}<0.05)$. Therefore, non-parametric inferential tests were used, namely the Spearman's rank correlation coefficient to measure relationships between the above variables, and the Kruskal-Wallis one-way analysis of variance to assess statistical significance of HSUV differences between disease groups. Finally, outcomes in each EQ5D-5L domain were treated as ordinal variables and therefore the Mann-Whitney U test was used to assess the statistical significance of differences in the EQ-5D-5L domain outcomes between disease areas. 


\section{Information and main variables of interest}

As not all participating countries had available EQ-5D-5L value sets at the time of analysis, the "EQ-5D-5L Crosswalk Index Value Calculator" was used to calculate utility scores based on the reported health states [11]. Subsequently, in order to obtain participants' utility loss, their utility scores were deducted from the general population Health State Utility Value (HSUV) of their respective countries of origin (where such a value was available) [12]. For participants whose country of origin did not yet have EQ-5D value sets, UK value sets were used as a proxy to calculate their utility score and respective utility loss.

In addition, patients' self-perceived QoL was measured using the EQ-5D-5L VAS tool consisting of a vertical $20-\mathrm{cm}$ scale rated from $0-100$, where 0 and 100 reflect the worst and the best imaginary health states, respectively [13]; respondents were asked to indicate a point on the scale that best represented their self-perceived overall health on the day the questionnaire was completed. We also measured disability level using the Barthel Index, a commonly used instrument for the evaluation of an individual's dependence level, based upon ability to perform 10 basic Activities of Daily Living (ADLs) [14]; a score of 20 shows complete independence, with 15-19 indicating mild dependence, 10-14 moderate dependence and 0-9 complete dependence due to disability. Finally, participants were asked to rate their satisfaction with the treatment they had received from the national healthcare system in their countries on a scale from 0 (not satisfied at all) to 10 (extremely satisfied). 


\section{Results}

\section{Sample size and demographics}

Out of 180 patient associations invited in 48 countries, 675 completed questionnaires eligible for analysis surveys were received from 32 countries. Countries comprising the largest parts of the returned sample included UK ( $n=351,52 \%$ of all sample), France ( $n=106,15.7 \%)$, USA $(n=78,11.6 \%)$ and Romania $(n=60,8.8 \%)$.

In terms of the condition suffered, our sample comprised of MS ( $\mathrm{n}=254,37.6 \%$ of total sample), BC ( $\mathrm{n}=179,26.5 \%), \mathrm{RD}(\mathrm{n}=140,20.7 \%), \mathrm{RA}(\mathrm{n}=53,7.8 \%)$ and $\mathrm{RC}(\mathrm{n}=49,7.2 \%)$ patients (a detailed list of RD and RC diagnoses is provided in Supplementary Table 1). Average patient age was $47( \pm 12.8)$ years and the majority of patients were females $(85 \%)$, married/cohabiting (66\%) and employed (42\%) (Table 1).

\section{Sample HRQoL}

\section{EQ-5D-5L Utility (HSUV)}

Table 2 describes the main findings related to the HRQoL outcomes of our sample. Average patient HSUV was $0.6( \pm 0.26)$, translating into an average health state utility loss of $29.4 \%$, when compared to the HSUVs identified for the general population in the study countries. Among disease areas, the lowest average HSUV (i.e. 0.46, \pm 0.31 ) was reported by RD, followed by MS $(0.56, \pm 0.27)$ and RA $(0.58, \pm 0.2)$ patient groups $(53.5 \%, 32.5 \%$ and $33 \%$ utility loss respectively), whereas the highest (i.e. $0.7, \pm 0.18$ ) was observed for both $\mathrm{BC}$ and RC patient groups (Table 2). 
A Kruskal-Wallis $H$ test showed that there were statistically significant differences $(p<0.001)$ in mean HSUV $\left(\chi^{2}=76.3\right)$ scores among disease areas (Table 2). More precisely, Mann Whitney U tests detected statistically significant differences in the HSUV mean rank of RD when compared to all other disease groups, namely $\mathrm{BC}(\mathrm{p}<0.001), \mathrm{RC}(\mathrm{p}<0.001), \mathrm{RA}$ $(\mathrm{p}=0.05)$ and $\mathrm{MS}(\mathrm{p}<0.05)$ (Table 3$)$, and of RA and MS when compared to $\mathrm{BC}(\mathrm{p}<0.001$ and $\mathrm{p}=0.001$ respectively) and $\mathrm{RC}(\mathrm{p}<0.001$ and $\mathrm{p}=0.001$ respectively). No statistically significant difference was observed in HSUV outcomes between BC and RC $(p=0.941)$ and between MS and RA ( $p=0.776)$. Finally, floor and ceiling effects existed in our sample $(n=24,3.6 \%$ and $\mathrm{n}=29,4.4 \%$ respectively), with lowest (negative) scores being more commonly reported by RD ( $n=12,9 \%)$ and MS ( $n=11,4 \%)$ patients and perfect health scores primarily observed for $\mathrm{BC}(6 \%, \mathrm{n}=10)$ patients.

\section{$<$ Table 1 about here $>$}

\section{$E Q-5 D-5 L$ VAS}

Our sample had an average EQ-5D-5L VAS score of $63( \pm 22)$ and this was moderately correlated to the EQ-5D-5L utility score (Spearman's rho=0.582, $\mathrm{p}<0.001$ ). Disease-specific EQ-5D-5L VAS outcomes reflected disease-specific EQ-5D-5L outcomes, with RD exhibiting the lowest VAS score, followed by MS, RA, RC and BC (Table 2). A KruskalWallis $H$ test showed that there were statistically significant differences $(p<0.001)$ in mean $\operatorname{VAS}\left(\chi^{2}=39.2\right)$ scores among disease areas (Table 2$)$. 
More precisely, Mann Whitney U tests detected statistically significant differences in the VAS mean rank of RD when compared to all other disease groups, namely $\mathrm{BC}(\mathrm{p}<0.001), \mathrm{RC}$ $(p=0.001), R A(p=0.001)$ and $M S(p<0.001)$, and of MS when compared to $B C(p<0.05)$.

\section{Barthel Index}

Half of the respondents $(50.5 \%)$ reported being independent on all ADLs indicating that, on average, our sample was only mildly dependent when it came to carrying out daily activities, whereas $\mathrm{BC}$ and $\mathrm{RC}$ patient groups were independent on all ADLs. A strong correlation was found between patient dependence level and the respective HSUV (Spearman's rho=0.591, $\mathrm{p}<0.001)$. A Kruskal-Wallis $\mathrm{H}$ test showed that there were statistically significant differences

$(\mathrm{p}<0.001)$ in mean BI $\left(\chi^{2}=118.2\right)$ scores among disease areas (Table 2). More precisely, Mann Whitney U tests detected statistically significant differences in the BI mean rank of RD when compared to all other disease groups, namely BC $(\mathrm{p}<0.001), \mathrm{RC}(\mathrm{p}<0.001), \mathrm{RA}$ $(\mathrm{p}<0.05)$ and MS $(\mathrm{p}<0.05)$ and, of RA and MS when compared to all other disease areas, namely $\mathrm{BC}(\mathrm{p}<0.001)$ and $\mathrm{RC}(\mathrm{p}<0.05$ and $\mathrm{p}<0.001$ respectively) but not when compared to each other $(p=0.445)$. Finally, it was observed that the BI score was the only HRQoL characteristic where outcomes between $\mathrm{RC}$ and $\mathrm{BC}$ had a statistically significant difference $(\mathrm{p}<0.05)$ 


\section{Decomposition of EQ-5D-5L}

Health profiles were examined to assess the proportions of patients across each disease area who reported at least some problems at each level on the EQ-5D-5L dimensions (Figure 1).

Decomposition of the EQ-5D-5L scores showed that overall, QoL problems varied between the EQ-5D dimensions and a worsening of HRQoL was brought about by problems of all severity levels (slight, moderate, severe, extreme) in the domains of "pain/discomfort" (82\%, of which $14 \%$ severe/extreme), followed by performing "usual activities" ( $75 \%$, of which $15 \%$ severe/extreme), “anxiety/depression" (70\%, of which $10 \%$ severe/extreme) and "mobility" (66\%, of which 15\% severe/extreme). Self-care was the least problematic EQ-5D5L domain across all sample and all disease areas $(39 \%$ of respondents reported problems, of which 7\% were severe/extreme).

Additionally, it was observed that EQ-5D-5L domains with the highest percentage of "severe and/or extreme" problems reported in each disease area included; anxiety/depression for BC patients (7\%), performing usual activities for $\mathrm{RD}$ and $\mathrm{RC}$ patients $(34 \%$ and $13 \%$ respectively), pain/discomfort for RA patients (13\%) and mobility for MS (18\%) (Figure 1). Finally, no statistically significant differences were observed when the outcomes of each disease group in the domain of "Anxiety/Depression" were compared to each other.

\section{$<$ Figure 1 about here $>$}

Assessment of disease-specific outcomes within EQ-5D-5L domains showed that RD had the highest mean scores (i.e. highest level of problems) in each domain (Table 3) and consequently yielded the greatest differences when compared to all other disease areas, across all domains. 
More precisely, it was demonstrated that these differences were all statistically significant (either at the $1 \%$ or $5 \%$ significance level), apart from those between RD and all other disease areas in "Anxiety/Depression" and those between RD and RA in the domains of "Self-care" and "Pain/Discomfort" (Table 3).

\section{$<$ Table 3 about here $>$}

However, with regards to RD patients poorer health outcomes in some domains relative to others were observed, with pain/discomfort having the greatest contribution towards worsening of QoL compared to the other disease groups studied (32\% compared to, for example, a range of $4 \%-13 \%$ of severe and/or extreme problems reported by other disease groups as shown on Figure 1).

Following RD, highest overall burden in all domains was observed for RA and MS groups, which both yielded statistically significant differences in "Mobility" $(p<0.05$ and $p<0.001$ respectively), "Self-Care" ( $p<0.001)$, "Usual activities" ( $p=0.001$ and $p<0.001$ respectively) and "Pain/Discomfort" ( $p<0.001)$ when compared to BC, in "Mobility" ( $p<0.05$ and $p<0.001$ respectively) and "Pain/Discomfort" $(\mathrm{p}<0.001)$ when compared to RC and only in "Pain/Discomfort" $(\mathrm{p}<0.05)$ when compared to each other.

In addition, a statistically significant difference $(\mathrm{p}<0.001)$ in mean scores existed between $B C$ and RC only in the domain of "Self-Care", although discrepancies were observed both in terms of severity and amount of problems reported in each EQ-5D-5L domain (Figure 1). For example: the domain of "Mobility" was the second, least problematic domain for both BC and RC patients, although the severity level reported by RC patients was higher compared to that reported by $\mathrm{BC}$ patients (i.e. $2 \%$ and $11 \%$ severe/extreme problems respectively). 
Similarly, even though both groups were equally problem free in the domain of "Usual activities" (i.e. $36 \%$ of $\mathrm{BC}$ and $34 \%$ of RC), the severe/extreme problems experienced in this domain by $\mathrm{RC}$ patients were much higher compared to $\mathrm{BC}$ (i.e. $13 \%$ compared to $5 \%$ respectively). On the contrary, in the domain of "Anxiety/Depression" both groups reported similar levels of severe/extreme problems (i.e. $7 \%$ for $\mathrm{BC}$ and $6 \%$ for $\mathrm{RC}$ ) but overall, only $27 \%$ of $\mathrm{BC}$ patients were problem free in this domain compared to $37 \%$ of $\mathrm{RC}$ patients.

\section{Discussion}

\section{HRQoL outcomes and differences between disease areas}

In this study we used a preference based HRQoL measurement tool to detect differences on HRQoL outcomes for five different diagnoses across three distinct therapeutic areas, namely $\mathrm{RD}$, Cancer (including BC and RC) and autoimmune diseases (including RA and MS).

Previous studies have assessed the patterns that differentiate HRQoL outcomes between the general population and patients $[15,16]$. However, among patient populations, it is still unclear how, the patterns that lead to HRQoL impairment are differentiated between, or even within (i.e. in different cancer diagnoses), therapeutic areas. Existing comparisons of HRQoL outcomes between disease-specific patient populations are complicated by differences in the various study designs and the instruments used for HRQoL measurement.

Furthermore, some of these studies are primarily focused on mapping outcomes generated by disease specific tools on generic measures, such as the EQ-5D. For example, a study on HRQoL outcomes between RA, MS and two types of cancer has mapped outcomes of disease specific tools on the EQ-5D and demonstrated results comparable to ours across disease areas, although as different clinical criteria were measured by each disease-specific tool, comparisons of HRQoL outcomes on the same end points were not feasible [17]. 
A further strength of our study is that we assessed the domains that differentiate HRQoL outcomes between RD and more common diseases. Various sources in the literature have presented the socioeconomic burden of individual RDs and these have demonstrated similarly low HSUV index scores [18-20]. However, only a few studies have provided comparisons between a range of RD and the general population $[21,22]$ and these only assessed outcomes in a limited number of RD diagnoses compared to ours, whereas only the latter also provided comparisons with common chronic diseases [22]. Nevertheless, these studies presented QoL outcomes as overall EQ-5D index scores derived from the literature, and therefore direct comparisons on the specific EQ-5D dimensions that differentiated HRQoL impairment between the disease groups were not provided. Our study being the only one to date to provide evidence from such a wide variety of RD diagnoses from an international population, contributes significantly to the literature in providing a holistic understanding of the dimensions that drive poor HRQoL outcomes in RD sufferers as a distinct therapeutic group. Essentially, this allows us to define more accurately those QoL considerations that should drive value in the health technology assessments of new therapies targeting rare as opposed to more common disease areas. Furthermore, a key feature of our study is that we have also provided comparisons between RC and other RDs (i.e. excluding cancer related RDs). Our findings show a clear improvement on HRQoL outcomes of RC compared to RD patients, highlighting the remaining unmet need in treating RDs as opposed to RCs - for which a significant number of therapies exists [23]. More importantly it highlights that "rarity of the condition targeted by a new therapy" itself would not necessarily add true value to the respective technology. This is all the more important when considering that orphan drugs, despite being unlikely to demonstrate cost-effectiveness, they are still more likely to receive a higher level of acceptance for reimbursement compared to those therapies targeting more prevalent conditions. [24]. 
Finally, another strength of our study is that we compared HRQoL outcomes between highly related therapeutic areas in terms of treatment and pathophysiological symptoms. More specifically, we observed that RA and MS groups experienced identical burden in terms of HSUVs and respective utility losses. Comparable utilities between the two conditions were expected since they both belong under the umbrella of autoimmune disorders and both are characterized by overlapping Musculoskeletal (MSK) related pathophysiological similarities, such as progressive joint damage, daily pain and functional impairment [25]. Nevertheless, we also detected discrepancies in HRQoL preferences between RA and MS. Although studies show a severe impact of pain on QoL deterioration of MSK disease sufferers [26], our findings show that problems with pain were valued differently between RA and MS patients. Indeed, evidence from the literature suggests that psychological factors play a major role in the pain and disability associated with arthritis [27, 28], while, in contrast, pain and anxiety/depression have been repeatedly found to be of less importance for MS cohorts, with Mobility typically reported as the most debilitating symptom [29]. Understanding the dimensions that are mostly impaired across diseases with pathophysiological similarities and hence, often with similar treatments (i.e. Tumor necrosis factor- $\alpha$ (TNF- $\alpha$ ) blockers for RA and MS [30]) is even more compelling when taking into account the growing trend among payers towards 'value and indication based pricing' whereby one product can have different prices per indication according to the outcomes it achieves for patient groups across the respective indications [24]. 


\section{Limitations and areas for further research}

We demonstrated significant differences in the dimensions that drive HRQoL outcomes between rare and more common disease areas and more importantly we showcased that the same EQ-5D utility may reflect very different severities depending on the patient population under investigation. We believe that the analysis we have presented will be valuable to improving future QoL research and Health Technology Assessment processes, as it has provided direct comparisons of disease-specific EQ-5D outcomes and hence could inform reimbursement decisions on interventions between and within therapeutic areas. However, out study is not without limitations. The first limitation relates to sampling issues; due to time constraints, a convenience patient sample was drawn, which mostly comprised Europeanbased patients. In addition, since our survey was only addressed to individuals across a limited number of chronic conditions, we acknowledge that, despite the sufficiently large overall sample size and the diversity of conditions studied, our results might still not be representative of the true magnitude of disease-specific HRQoL differences existing in an international population of chronically ill individuals; this can be especially true when considering that that the majority of disease areas studied here are predominantly manifested in female populations (i.e. $\mathrm{BC}$, RA and MS) and as such resulted in a disproportionately higher female population included in our study. Furthermore, the use of a web-survey itself raises issues related to the interpretation of the survey questions by the respondents, the levels of understanding of the different EQ-5D-5L domains and the respective severity levels described. There are also methodological issues associated with the utility and utility loss calculations. Firstly, according to table 1, there is an average of 9 years between patients' diagnosis and the moment they responded to the survey; this implies that for curable conditions such as $\mathrm{BC}$ and $\mathrm{RC}$, some of the respondents would have already been cured or not necessarily receiving treatment at the moment of completing the survey. 
However, we still considered the contribution of these individuals as important and relevant to our research. Due to the effects of cancer and its treatment, cancer survivors often experience physical problems such as pain, fatigue, or musculoskeletal problems, even years after diagnosis and hence, they have repeatedly been shown to have a reduced physical and mental HRQoL compared with general population samples [31]. Nevertheless, to improve accuracy of the results a future replication of this research should distinguish between cancer survivors (previously treated for cancer) and those that are receiving treatment for cancer at the time they are completing the EQ-5D-5L. Secondly, due to time and data constraints utility loss calculations were not adjusted for age, gender or other co-morbidities, introducing concerns for potential over/under estimation of our findings. As such our findings should be interpreted with caution and future replication of the study should take such adjustments into account. Thirdly, using UK-specific population norms as a proxy to determine the health disutility of participants from countries which had no population norms reported in the literature is another limitation in our study. However, as these countries represented less than $20 \%$ of our sample we did not expect this limitation to have a significant impact on the overall results. Indeed, a quasi-robustness check (Supplementary Table 2) performed using scores from countries that comprised the largest part (nearly $80 \%$ ) of our sample and for which national population norms existed (namely the UK, USA and France) highly reflected the findings presented in Table 3 , showcasing that regardless of country values used, the health states exhibited by individuals in the study disease areas were still differentiated following similar patterns. Finally, utilisation of a generic tool such as the EQ-5D has often been proven inadequate in capturing a critical domain, which largely shapes QoL outcomes in particular disease areas [32]. Therefore, we suggest that future research should also assess how patient groups are differentiated based on the outcomes of these key QoL domains not included in a generic, preference based tool. 


\section{Conclusion}

Increasingly stretched health care budgets, and associated challenges in healthcare decision making, necessitate that health systems worldwide invest in services and interventions which achieve the most meaningful outcomes across disease areas, in the most efficient way. This implies that care needs to be tailored such that it would improve those disease specific dimensions that would provide the greatest benefit for patients. Despite the limitations of our study, the comparisons presented here are worthy of note, since they contribute in our understanding of the drivers of impaired HRQoL in one disease area relative to another. Ultimately, the findings of these comparisons can be used to inform decisions about the optimal allocation of resources based on patient groups with those chronic diseases who are in greatest need. Finally, the particular worsening of QoL demonstrated for RDs compared to other chronic disease areas, and even more importantly compared to RC highlights that RDs still present with significant unmet need in terms of therapies and remain an issue of high priority not only for health policy makers and the industry but also for patients and patient advocacy groups themselves. 


\section{Acknowledgements}

We are grateful for the invaluable support of all the European and international patient associations that were invited to participate in the study and voluntarily agreed to share the web-survey links with their networks of patients. Finally, we are thankful to Hala Hourani, Ansgar Lange, Erica Visintin and Olivier Wouters for their assistance in translating the survey questionnaires and for providing valuable support in the research process.

\section{Source of funding}

This study was supported by Advance-HTA, a research grant that has received funding from the European Commission, DG Research, $7^{\text {th }}$ Framework Programme for Research (grant agreement No. 305983). The views expressed in this study are those of the authors and do not represent the views of the European Commission, DG Research. 


\section{References}

1. Schlenk E.A., Erlen J.A., Dunbar-Jacob J., McDowell J., Engberg S., Sereika S.M., Rohay J.M., Bernier M.J. Health-related quality of life in chronic disorders: a comparison across studies using the MOS SF-36. Qual Life Res. 1998 Jan;7(1):57-65.

2. Global Burden of Disease Cancer Collaboration. The Global Burden of Cancer 2013. JAMA oncology. 2015;1(4):505-527.

3. Global Alliance for Musculoskeletal Health. 2010. Influential global alliance calls on governments and the World Health Organisation to prioritise musculoskeletal health following findings of Global Burden of Disease Study. Available online at: http://bjdonline.org/musculoskeletal-conditions-the-second-greatest-cause-of-disability-2/ (Accessed April 10, 2018).

4. Pfizer, Value of Medicines. Value of medicines for rare diseases. Issued by Global Policy and International Public Affairs. Available online at: http://www.pfizer.com/files/health/Value of Medicine Rare Diseases.pdf (Accessed April 22, 2018).

5. Brazier J. \& Rowen D. (2011). NICEDSU TECHNICALSUPPORT DOCUMENT 11: ALTERNATIVES TO EQ-5D FOR GENERATING HEALTH STATE UTILITY VALUES. REPORT BY THE DECISION SUPPORT UNIT. March 2011. Available online at; http://www.nicedsu.org.uk/TSD11\%20Alternatives $\% 20$ to $\% 20 \mathrm{EQ}-5 \mathrm{D}$ final.pdf (Accessed April 6, 2016).

6. EURORDIS (2010). Why Research on Rare Diseases? Available online at: http://www.eurordis.org/sites/default/files/publications/why rare disease_research.pdf (Accessed March 28, 2016) 
7. Schuller Y., Hollak C.E.M., Biegstraaten M. The quality of economic evaluations of ultra-orphan drugs in Europe - a systematic review. Orphanet Journal of Rare Diseases. $2015 ; 10: 92$.

8. Marra C.A., Woolcott J.C., Kopec J.A., Shojania K., Offer R., Brazier J.E., Esdaile J.M., Anis A.H. A comparison of generic, indirect utility measures (the HUI2, HUI3, SF-6D, and the EQ-5D) and disease-specific instruments (the RAQoL and the HAQ) in rheumatoid arthritis. SocSci Med. 2005 Apr; 60(7):1571-82.

9. Sprangers M.A., de Regt E.B., Andries F., van Agt H.M., Bijl R.V., de Boer J.B., Foets M., Hoeymans N., Jacobs A.E., Kempen G.I., Miedema H.S., Tijhuis M.A., de Haes H.C. Which chronic conditions are associated with better or poorer quality of life? J Clin Epidemiol. 2000 Sep;53(9):895-907.

10. EuroQol. Who is using EQ-5D?. Available online at: http://www.euroqol.org/about-eq5d/how-to-use-eq-5d/who-is-using-eq-5d.html (Accessed March 9, 2015).

11. EuroQoL. EQ-5D-5L, Valuation. Available online at: https://euroqol.org/eq-5dinstruments/eq-5d-51-about/valuation/ (Accessed March 11, 2015)

12. EuroQoL. EQ-5D-5L, Population Norms. Available online at: https://euroqol.org/eq-5dinstruments/eq-5d-31-about/population-norms/ (Accessed March 11, 2015).

13. Brooks R.; EuroQoL Group. EuroQoL: the current state of play. Health Policy 1996; 37:53-72.

14. Angelis A., Kanavos P., López-Bastida J., Linertová R., Nicod E., Serrano-Aguilar P. and BURQOL-RD Research Network. Social and economic costs and health-related quality of life in non-institutionalised patients with cystic fibrosis in the United Kingdom. BMC Health Services Research 2015 15:428.

15. De Wit G.A., Busschbach J.J., De Charro F.T. Sensitivity and perspective in the valuation of health status: whose values count? Health Econ. 2000 Mar; 9(2):109-26. 
16. Krabbe P.F., Tromp N., Ruers T.J., van Riel P.L. Are patients' judgments of health status really different from the general population? Health Qual Life Outcomes. 2011 May 11; $9: 31$.

17. Versteegh M.M., Leunis A., Uyl-de Groot C.A., Stolk E.A. Condition-specific preference-based measures: benefit or burden? Value Health. 2012 May; 15(3):504-13.

18. López-Bastida J., Linertová R., Oliva-Moreno J., Posada-de-la-Paz M., Serrano-Aguilar P., Kanavos P., Taruscio D., Schieppati A., Iskrov G., Baji P., Delgado C., von der Schulenburg J.M., Persson U., Chevreul K., Fattore G.; BURQOL-RD Research Network. Social/economic costs and health-related quality of life in patients with PraderWilli syndrome in Europe. Eur J Health Econ. 2016 Apr; 17Suppl 1:99-108.

19. Chevreul K., Gandré C., Brigham K.B., López-Bastida J., Linertová R., Oliva-Moreno J., Serrano-Aguilar P., Posada-de-la-Paz M., Taruscio D., Schieppati A., Iskrov G., Gulácsi L., von der Schulenburg J.M., Kanavos P., Persson U., Fattore G.; BURQOL-RD Research Network. Social/economic costs and health-related quality of life in patients with fragile X syndrome in Europe.Eur J Health Econ. 2016 Apr; 17Suppl 1:43-52.

20. Cavazza M., Kodra Y., Armeni P., De Santis M., López-Bastida J., Linertová R., OlivaMoreno J., Serrano-Aguilar P., Posada-de-la-Paz M., Taruscio D., Schieppati A., Iskrov G., Péntek M., von der Schulenburg J.M., Kanavos P., Chevreul K.,Persson U., Fattore G.; BURQOL-RD Research Network. Social/economic costs and health-related quality of life in patients with Duchenne muscular dystrophy in Europe. Eur J Health Econ. 2016 Apr; 17Suppl 1:19-29.

21. Winter Y., Schepelmann K., Spottke A.E., Claus D., Grothe C., Schröder R., Heuss D., Vielhaber S., Tackenberg B., Mylius V., Reese J.P., Kiefer R., Schrank B., Oertel W.H., Dodel R. Health-related quality of life in ALS, myasthenia gravis and facioscapulohumeral muscular dystrophy. J Neurol. 2010 Sep; 257(9):1473-81. 
22. Calvert M., Pall H., Hoppitt T., Eaton B., Savill E., Sackley C. Health-related quality of life and supportive care in patients with rare long-term neurological conditions. Qual Life Res. 2013 Aug; 22(6):1231-8.

23. Kanavos P \& Nicod E. What is wrong with orphan drug policies? Suggestions for ways forward. Value Health. 2012 Dec;15(8):1182-4.

24. Drummond M. \& Towse A. Orphan drugs policies: a suitable case for treatment. Eur J Health Econ. 2014 May;15(4):335-40.

25. Mpofu S., Moots R. A case of multiple sclerosis associated with rheumatoid arthritis and positive anticardiolipin antibodies. Annals of the Rheumatic Diseases. 2003; 62(4):376.

26. Dua T., Garrido Cumbrera M., Mathers C., Saxena S. WHO 2006. Neurological Disorders: Public Health Challenges. Chapter 2: Global burden of neurological disorders estimates and projections. (C) World Health Organization 2006. Available online at: http://www.who.int/mental_health/neurology/chapter_2 neuro_disorders_public h chall enges.pdf (Accessed July 10, 2016)

27. Dickens C., Creed F. The burden of depression in patients with rheumatoid arthritis. Rheumatology (Oxford). 2001 Dec;40(12):1327-30.

28. Hunter D.J., Riordan E.A. The impact of arthritis on pain and quality of life: an Australian survey. Int J Rheum Dis. 2014 Feb;17(2):149-55.

29. Sidovar M.F., Limone B.L., Coleman C.I. Mapping of Multiple Sclerosis Walking Scale (MSWS-12) to five-dimension EuroQol (EQ-5D) health outcomes: an independent validation in a randomized control cohort. Patient Relat Outcome Meas. 2016 Feb 3;7:138.

30. Kemanetzoglou, E. \& Andreadou, E. CNS Demyelination with TNF- $\alpha$ Blockers. Current Neurology and Neuroscience Reports. 2017. 17(4), 36. 
31. Heins M.J., Korevaar J.C., Hopman P.E., Donker G.A., Schellevis F.G., Rijken M.P. Health-related quality of life and health care use in cancer survivors compared with patients with chronic diseases. Cancer. 2016 Mar 15;122(6):962-70.

32. Tordrup D., Mossman J., Kanavos P. Responsiveness of the EQ-5D to clinical change: is the patient experience adequately represented? Int J Technol Assess Health Care. 2014 Jan; 30(1):10-19. 
Table 1. Sample employment and demographic characteristics

Note: $\mathrm{BC}=$ Breast Cancer, $\mathrm{RD}=$ Rare Diseases, $\mathrm{RC}=$ Rare Cancers, $\mathrm{RA}=\mathrm{Rheumatoid}$ Arthritis, MS=Multiple Sclerosis 
Table 2. Sample HRQoL characteristics [mean $(S D)]$ and statistical significance $(p)$ of differences among disease groups.

Note: $\mathrm{BC}=$ Breast Cancer, $\mathrm{RD}=$ Rare Diseases, $\mathrm{RC}=$ Rare Cancers, $\mathrm{RA}=$ Rheumatoid Arthritis, MS=Multiple Sclerosis 
Table 3. Mean scores of EQ-5D-5L domains and EQ-5D-5L HSUV across disease areas and statistical significance $(p)$ of differences between $\mathrm{RD}$ and other disease areas.

Note: $\mathrm{BC}=$ Breast Cancer, $\mathrm{RD}=$ Rare Diseases, $\mathrm{RC}=$ Rare Cancers, $\mathrm{RA}=$ Rheumatoid Arthritis, MS=Multiple Sclerosis 
Figure 1. Level of problems reported (\%) in each EQ-5D-5L domain across disease areas

Note: $\mathrm{BC}=$ Breast Cancer, $\mathrm{RD}=$ Rare Diseases, $\mathrm{RC}=$ Rare Cancers, $\mathrm{RA}=$ Rheumatoid Arthritis, MS=Multiple Sclerosis 

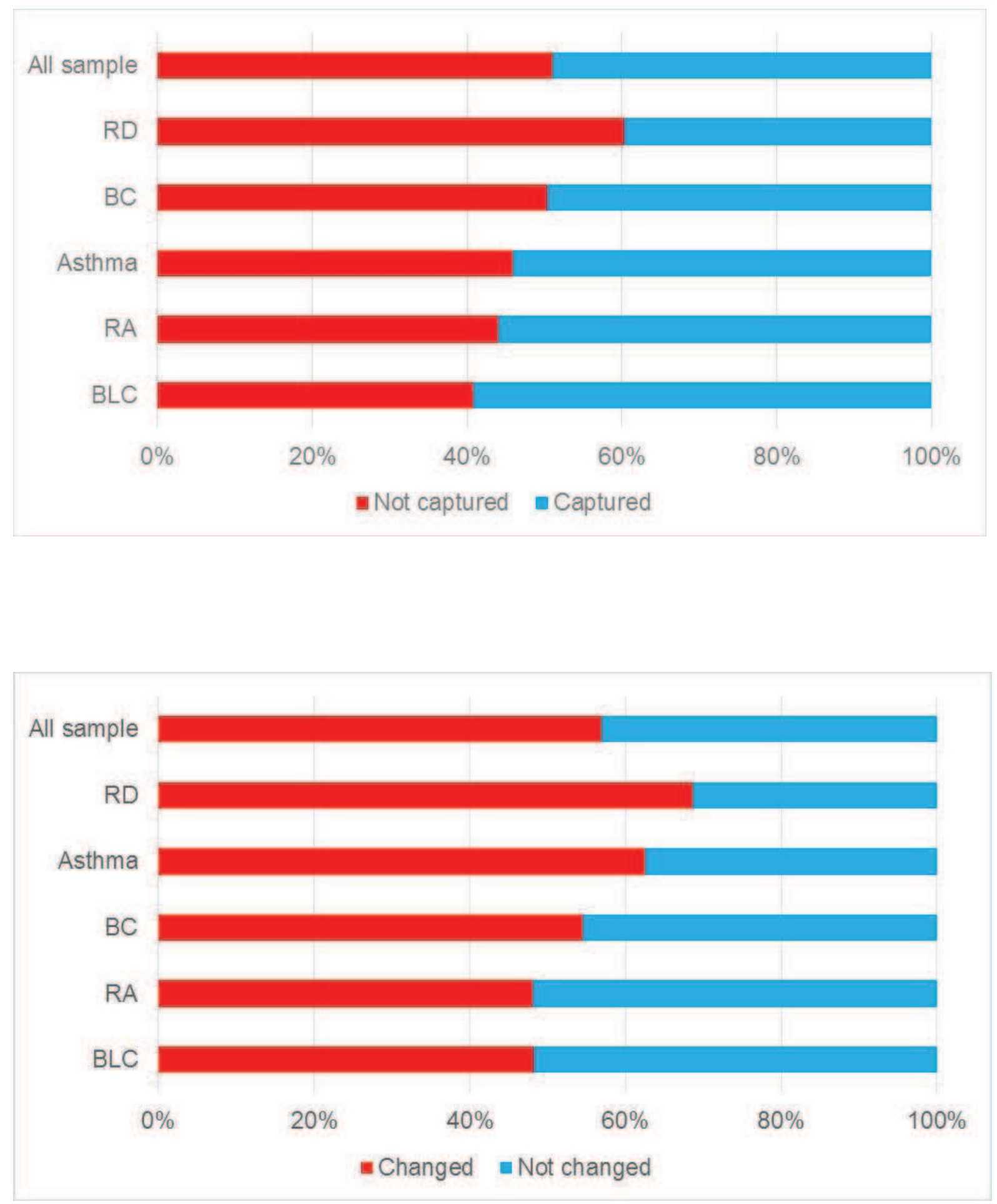